\title{
Contribuição da análise do seio maxilar para a identificação humana
}

\section{Contribution of the maxillary sinus analysis for human identification}

\author{
Jamilly de Oliveira Musse ${ }^{1}$, Jeidson Antônio Morais Marques², \\ Rogério Nogueira de Oliveira ${ }^{3}$
}

\begin{abstract}
Musse JO, Marques JAM, Olibeira RN. Contribuição da análise do seio maxilar para a identificação humana. Saúde, Ética \& Justiça. 2009;14(2):65-76.

Resumo: Os seios maxilares fornecem subsídios importantes para a identificação forense. Os objetivos deste estudo foram avaliar a possibilidade de investigação do sexo e de identificação humana pelos seios maxilares e propor três métodos de identificação. A amostra contou com 656 radiografias panorâmicas de 328 indivíduos, de ambos os sexos, divididos em grupo controle e experimental, segundo a ausência ou presença de perda dentária. Três métodos foram empregados: dois manuais e um computadorizado. Nas técnicas manuais, calculou-se a altura e largura dos seios, em folhas de acetato e avaliou-se a morfologia destes por quadrantes. Na computadorizada, foi calculada a área, perímetro e fator de forma, através do programa IMAGELAB $2000 \AA$. Em seguida, realizou-se a análise estatística e comparativa dos dados. A morfologia e as mensurações dos seios podem ser utilizadas para estimativa do sexo e identificação humana individual. A análise dos seios maxilares associada a outras evidências é passível de ser empregada nos Institutos Médico-Legais, podendo contribuir nos casos de identificação humana.
\end{abstract}

DESCRITORES: Antropologia forense; Seio maxilar; Radiografia panorâmica; Odontologia legal.

Professora do Curso de Odontologia da Universidade Estadual de Feira de Santana.

2 Professor Doutor do Curso de Odontologia da Universidade Estadual do Sudoeste da Bahia.

3 Professor Doutor da Faculdade de Odontologia da Universidade de São Paulo.

Endereço para correspondência: Avenida Franco Manuel da Silva, 437, Cidade Nova, Feira de Santana. CEP: 44053-060. E-mail: jamillymusse@ usp.br/musse_jo @ hotmail.com. 
INTRODUÇÃO

A identidade é o conjunto de caracteres que individualizam uma pessoa ou uma coisa, fazendo-a distinta das demais. É uma série de atributos que torna alguém ou alguma coisa igual apenas a si próprio. Identificar é determinar a individualidade, ou seja, é provar por meios técnicos e científicos que aquela pessoa é ela e não outra. É o ato mediante o qual se estabelece a identidade'.

A literatura relata que um processo de identificação humana deve atender a exigências biológicas, tais como a unicidade e imutabilidade das características analisadas, e requisitos técnicos como a classificabilidade e praticabilidade destes achados. Fundamentada nestes requisitos, a análise papiloscópica atualmente constitui a técnica de identificação humana mais praticada em todo o mundo. Entretanto, diante de situações nas quais os corpos vêm a óbito por catástrofes de grandes proporções, acidentes de tráfego, exposição prolongada a fatores ambientais ou por outros meios que alterem significativamente ou destruam os tecidos moles, a Antropologia Física e a Odontologia Legal ${ }^{21}$ podem ser utilizadas em conjunto, oferecendo maior confiabilidade aos resultados.

$\mathrm{Na}$ análise antropológica, o perito utiliza parâmetros estatísticos, associando características qualitativas e quantitativas a determinado grupo populacional na tentativa, principalmente, de estimar dados, como: espécie, sexo, fenótipo cor de pele, idade, estatura e peso. Neste caso, não se têm dados anteriores à morte do indivíduo e se procura o estabelecimento de características genéricas para uma identificação geral ${ }^{3}$.

Ao contrário da Antropologia, o emprego do exame odonto-legal para identificação humana depende da documentação produzida em momento anterior à época do exame e que sirva de base de confronto para a individualização do objeto periciado, tornando possível uma identificação personalista ou individual. ${ }^{4}$ Neste caso, dentre as técnicas utilizadas para alcançar o estabelecimento da identidade, a comparação radiográfica vem sendo empregada, há mais de 70 anos, possibilitando uma identificação cientificamente segura ${ }^{5}$.

Segundo Wood ${ }^{6}$, as radiografias mais utilizadas nas perícias odonto-legais de identificação humana são as do crânio, da face, dos ossos longos e dos dentes. As radiografias da face, por sua vez, permitem a análise de uma grande variedade de estruturas anatômicas, entre elas, os seios frontais e maxilares. A visualização dos contornos dessas estruturas, com figuras geométricas variadas, que se sobrepõem definem precisamente uma identidade.

Nesse sentido, diversos trabalhos empregam radiografias no processo de identificação humana, inclusive quando esta é obtida através dos seios da face. Entretanto, observa-se escassez de pesquisas que utilizem as radiografias odontológicas para estimativa de sexo e identificação humana individual, através dos seios maxilares ${ }^{7,9}$.

Perrella, et al. ${ }^{7}$ avaliaram a precisão e acurácia de medidas lineares da região de seio maxilar realizadas em filmes tomográficos, por meio de comparação com a reconstrução da imagem em 3D. Medidas lineares de ambos os seios maxilares foram realizadasemtomografiascomputadorizadas (2D-TC) de 17 pacientes, normais e com lesão, por dois examinadores, calibrados, separadamente, duas vezes cada um, utilizando um compasso. Um terceiro observador realizou as mesmas medidas eletronicamente na reconstrução 3D-TC. ANOVA (análise de variância) foi utilizada para a análise estatística. A porcentagem de erro intraexaminadores foi pequena em ambos os casos, com ou sem lesão, variando de $1,14 \%$ a 1,82\%. O erro inter-examinadores foi maior, alcançando o valor de $2,08 \%$. Os erros percentuais de acurácia nas amostras com lesão foram maiores do que os referentes às que não tinham lesão. A tomografia computadorizada em 2D-TC proporcionou precisão e acurácia adequadas para análise dos seios maxilares.

Fernandes $^{9}$ avaliou como a influência do volume, da forma e das dimensões dos seios maxilares, obtidos de radiografias de crânios secos, interferem na diferenciação do sexo e grupo étnico. A análise da função discriminante mostrou uma concordância de $90 \%$ para o grupo étnico e de $79 \%$ para o gênero.

O mesmo autor, em outro estudo, comparou 
o volume dos seios maxilares de crânios secos obtidos de cadáveres de descendência européia e Zulu, com seus respectivos grupos étnicos e gêneros. Os resultados mostraram que existem diferenças de volume dos seios maxilares entre as categorias estudadas ${ }^{8}$.

Teke et al. ${ }^{10}$ verificaram o potencial de investigação do sexo através do comprimento, da largura e da altura dos seios maxilares, medidos na tomografia computadorizada. Ao contrário do trabalho anterior, os resultados sugeriram baixa acurácia do método.

Nesse sentido, torna-se relevante a realização de trabalhos que testem a aplicabilidade da análise dos seios maxilares para estimativa de dados biotipológicos, como o sexo e para identificação humana individual.

\section{Objetivos}

Os objetivos deste estudo foram: avaliar a possibilidade de identificação humana individual e investigação do sexo, pelos seios maxilares e propor três métodos de identificação através das referidas estruturas. Isto se justifica a partir do momento em que se acredita que estas estruturas anatômicas variem de dimensão entre os indivíduos e se constata que inexiste uma padronização adequada de suas características, para possível arquivamento e posterior confronto.

\section{Material e mÉtodos}

Após aprovação pelo Comitê de Ética da Faculdade de Odontologia da Universidade de São Paulo (FOUSP) (Parecer 184566), os examinadores foram calibrados para mensuração de medidas dos seios maxilares (altura, largura, área, perímetro e fator de forma), a partir de radiografias panorâmicas. A concordância intra e inter-examinadores serviu para padronizar as mensurações e assegurar a reprodutibilidade da técnica.

A amostra contou com 656 radiografias panorâmicas, correspondentes à 328 indivíduos (164 de cada gênero), adultos, com idade superior a 20 anos, sendo estes divididos em: Grupo I/controle: formado por radiografias de 164 pacientes ( 82 homens e 82 mulheres) submetidos a tratamento ortodôntico, mas que não necessitaram de exodontia de dentes posteriores e; Grupo II/ experimental: composto por radiografias de 164 pacientes (82 homens e 82 mulheres) submetidos a tratamento ortodôntico que necessitaram de exodontia de algum dente posterior.

De cada indivíduo, foram utilizadas duas radiografias, uma do início do tratamento e outra após dois anos de duração do tratamento ortodôntico. A partir daí, em cada radiografia, três métodos foram empregados, em ambos os grupos.

$\mathrm{Na}$ técnica manual I foi realizado o contorno dos seios maxilares direito e esquerdo, com uma folha de acetato sobre a radiografia panorâmica e, em seguida, procederam-se as mensurações, em milímetros, com o uso de uma régua.

Por falta de padronização de técnica manual na literatura, os pontos de referência das mensurações foram estabelecidos consultando especialistas em anatomia e radiologia e adaptando a metodologia preconizada por Ribeiro ${ }^{11}$ para o seio frontal.

Aproveitando o traçado da altura e largura máxima feito na transparência sobre a radiografia panorâmica, na técnica anteriormente citada, na técnica manual II, a cavidade aerada dos seios maxilares foi dividida em quatro quadrantes (Q1, Q2, Q3 e Q4) (Figura 1). A partir desta divisão, foi comparada a morfologia dos quadrantes através da sobreposição das folhas de acetato correspondentes às radiografias do mesmo indivíduo.

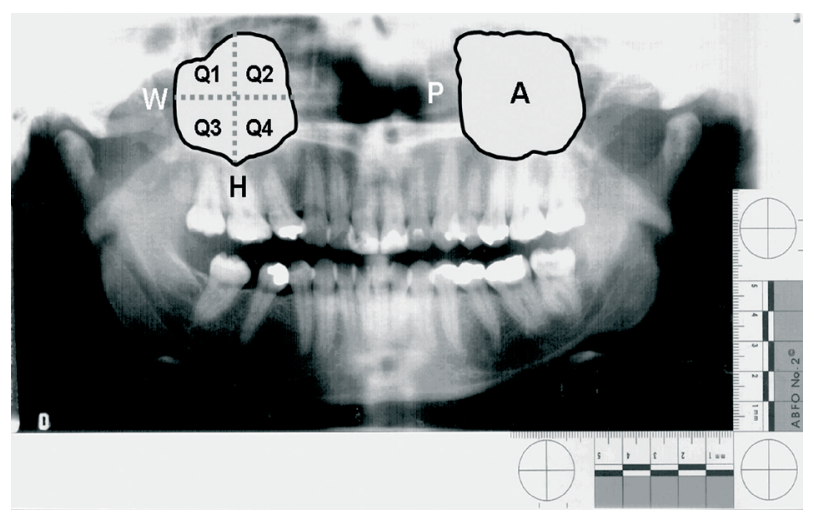

Figura 1. Pontos de referência utilizados na análise de medição, São Paulo, 2009

$\mathrm{Na}$ técnica computadorizada, após digitalização de todas as panorâmicas, os 
Musse JO, Marques JAM, Olibeira RN. Contribuição da análise do seio maxilar para a identificação humana

contornos dos seios foram realizados no computador e suas respectivas áreas $\mathrm{e}$ perímetro calculados, utilizando o software IMAGELAB 2000®. Além das medidas referidas anteriormente, outra mensuração foi empregada, o fator de forma. ${ }^{12}$ Trata-se de um valor, calculado através da relação entre a área e o perímetro, que expressa o quanto a morfologia dos seios maxilares foram preservadas na comparação das radiografias do mesmo indivíduo (Figura 1).

$\mathrm{Na}$ análise dos resultados, foram utilizadas técnicas da estatística descritiva (média e desvio padrão), teste t-Student com variâncias iguais ou desiguais e t-Student pareado para as variáveis quantitativas. O nível de significância utilizado nos testes estatísticos foi de 5,0\%. O software utilizado para a obtenção dos cálculos estatísticos foi o SPSS na versão 13. A análise entre os quadrantes foi feita através da comparação visual.

\section{Resultados}

Na técnica manual I, avaliou-se, separadamente, o resultado das mensurações na radiografia inicial (Tabela 1) e na após dois anos de tratamento (Tabela 2). Em ambas, para todas as variáveis, as médias foram mais elevadas no sexo masculino do que no feminino.

Tabela 1. Média e desvio padrão das medidas (altura e largura), segundo o sexo e o grupo na radiografia inicial, São Paulo, 2009

\begin{tabular}{|c|c|c|c|c|}
\hline Variável & Sexo & Grupo Controle | Média \pm DP & Grupo Experimental | Média \pm DP & Valor de $p$ \\
\hline Altura direita & Masculino & $43,59 \pm 6,46$ & $42,10 \pm 10,26$ & $\mathrm{p}^{(1)}=0,268$ \\
\hline (ARISD) & Feminino & $38,95 \pm 7,18$ & $37,51 \pm 7,90$ & $p^{(1)}=0,224$ \\
\hline \multicolumn{2}{|c|}{ Valor de $p$} & $\mathrm{p}^{(1)}<0,001^{*}$ & $p^{(1)}=0,002^{*}$ & \\
\hline Largura direita & Masculino & $48,83 \pm 8,65$ & $48,85 \pm 7,10$ & $\mathrm{p}^{(2)}=0,984$ \\
\hline (LRISD) & Feminino & $46,02 \pm 6,49$ & $45,51 \pm 6,91$ & $p^{(1)}=0,625$ \\
\hline \multicolumn{2}{|c|}{ Valor de $p$} & $\mathrm{p}^{(2)}=0,020^{*}$ & $p^{(1)}=0,003^{*}$ & \\
\hline Altura esquerda & Masculino & $44,34 \pm 9,54$ & $43,22 \pm 8,46$ & $\mathrm{p}^{(1)}=0,427$ \\
\hline (ARISE) & Feminino & $40,61 \pm 7,47$ & $42,56 \pm 7,61$ & $\mathrm{p}^{(1)}=0,099$ \\
\hline \multicolumn{2}{|c|}{ Valor de $p$} & $\mathrm{p}^{(1)}=0,006^{*}$ & $\mathrm{p}^{(1)}=0,601$ & \\
\hline Largura esquerda & Masculino & $50,41 \pm 7,61$ & $49,56 \pm 6,36$ & $\mathrm{p}^{(1)}=0,437$ \\
\hline (LRISE) & Feminino & $48,73 \pm 6,85$ & $46,78 \pm 6,55$ & $p^{(1)}=0,064$ \\
\hline \multicolumn{2}{|c|}{ Valor de $p$} & $\mathrm{p}^{(1)}=0,139$ & $p^{(1)}=0,007^{*}$ & \\
\hline
\end{tabular}

${ }^{*}$ ): Diferença significante a 5,0\%. (1): Através do teste t-Student com variâncias iguais. (2): Através do teste t-Student com variâncias desiguais.

Tabela 2. Média e desvio padrão das medidas (altura e largura), segundo o sexo e o grupo na radiografia após dois anos de tratamento, São Paulo, 2009

\begin{tabular}{|c|c|c|c|c|}
\hline Variável & Sexo & Grupo Controle | Média \pm DP & Grupo Experimental | Média \pm DP & Valor de $p$ \\
\hline Altura direita & Masculino & $43,54 \pm 6,54$ & $42,41 \pm 9,66$ & $\mathrm{p}^{(1)}=0,385$ \\
\hline (ARISD) & Feminino & $39,61 \pm 8,74$ & $37,24 \pm 7,61$ & $\mathrm{p}^{(2)}=0,066$ \\
\hline \multicolumn{2}{|c|}{ Valor de $p$} & $\mathrm{p}^{(1)}=0,001^{*}$ & $\mathrm{p}^{(2)}<0,001^{*}$ & \\
\hline Largura direita & Masculino & $49,56 \pm 7,59$ & $47,41 \pm 6,87$ & $\mathrm{p}^{(2)}=0,059$ \\
\hline (LRISD) & Feminino & $46,76 \pm 6,35$ & $42,88 \pm 7,00$ & $\mathrm{p}^{(2)}<0,001^{\star}$ \\
\hline \multicolumn{2}{|c|}{ Valor de $p$} & $\mathrm{p}^{(2)}=0,011^{*}$ & $\mathrm{p}^{(2)}<0,001^{*}$ & \\
\hline Altura esquerda & Masculino & $44,34 \pm 8,20$ & $44,05 \pm 9,30$ & $\mathrm{p}^{(1)}=0,831$ \\
\hline (ARISE) & Feminino & $40,27 \pm 6,89$ & $42,07 \pm 7,29$ & $\mathrm{p}^{(2)}=0,105$ \\
\hline \multicolumn{2}{|c|}{ Valor de $p$} & $\mathrm{p}^{(2)}=0,001^{*}$ & $\mathrm{p}^{(1)}=0,132$ & \\
\hline Largura esquerda & Masculino & $51,51 \pm 7,95$ & $49,34 \pm 6,31$ & $p^{(1)}=0,055$ \\
\hline (LRISE) & Feminino & $48,73 \pm 6,28$ & $43,98 \pm 7,21$ & $\mathrm{p}^{(2)}<0,001^{*}$ \\
\hline \multicolumn{2}{|c|}{ Valor de $p$} & $\mathrm{p}^{(1)}=0,014^{*}$ & $\mathrm{p}^{(2)}<0,001^{*}$ & \\
\hline
\end{tabular}

${ }^{*}$ ): Diferença significante a 5,0\%. (1): Através do teste t-Student com variâncias desiguais. (2): Através do teste t-Student com variâncias iguais. 
$\mathrm{Na}$ radiografia inicial, observou-se diferença significante, entre os sexos, exceto para as variáveis "largura esquerda" no grupo controle e "altura esquerda" no grupo experimental. Entre os grupos (controle e experimental) não foi observada diferença significante.

$\mathrm{Na}$ final, comprovou-se diferença entre os sexos, com exceção da variável altura esquerda no grupo experimental. $\mathrm{Na}$ comparação entre os grupos, observou-se diferença significativa entre os mesmos para as variáveis: "Largura direita" e "Largura esquerda" no sexo feminino.
A Tabela 3 apresenta a média e o desvio padrão das variações entre os valores encontrados na radiografia inicial e final, por sexo e por grupo. Entre os sexos, a única diferença significante foi registrada na variável Largura esquerda do grupo experimental. Diferenças entre os grupos foram verificadas para "largura direita", em ambos os sexos e "largura esquerda", no sexo feminino. Para estas variáveis, observou-se que as médias foram positivas no grupo experimental e negativas no grupo controle, exceto para "largura esquerda" no sexo feminino.

Na comparação da morfologia dos quadrantes

Tabela 3. Média e desvio padrão das diferenças (variação) entre as medidas (altura e largura) da radiografia inicial e após dois anos de tratamento, segundo o sexo e o grupo, São Paulo, 2009

\begin{tabular}{|c|c|c|c|c|}
\hline Variável & Sexo & Grupo Controle | Média \pm DP & Grupo Experimental | Média \pm DP & Valor de $p(1)$ \\
\hline Altura direita & Masculino & $0,05 \pm 5,59$ & $-0,32 \pm 9,87$ & $\mathrm{p}^{(1)}=0,771$ \\
\hline (ARISD) & Feminino & $-0,66 \pm 6,22$ & $0,27 \pm 3,62$ & $\mathrm{p}^{(2)}=0,245$ \\
\hline \multicolumn{2}{|c|}{ Valor de $p$} & $\mathrm{p}^{(1)}=0,445$ & $\mathrm{p}^{(2)}=0,615$ & \\
\hline Largura direita & Masculino & $-0,73 \pm 6,95$ & $1,44 \pm 4,67$ & $p^{(2)}=0,020^{*}$ \\
\hline (LRISD) & Feminino & $-0,73 \pm 6,96$ & $2,63 \pm 4,68$ & $\mathrm{p}^{(2)}<0,001^{*}$ \\
\hline \multicolumn{2}{|c|}{ Valor de $p$} & $\mathrm{p}^{(1)}=1,000$ & $\mathrm{p}^{(1)}=0,104$ & \\
\hline Altura esquerda & Masculino & $0,00 \pm 8,70$ & $-0,83 \pm 4,73$ & $\mathrm{p}^{(2)}=0,450$ \\
\hline (ARISE) & Feminino & $0,34 \pm 3,81$ & $0,49 \pm 3,84$ & $\mathrm{p}^{(1)}=0,807$ \\
\hline \multicolumn{2}{|c|}{ Valor de $p$} & $\mathrm{p}^{(2)}=0,745$ & $\mathrm{p}^{(1)}=0,052$ & \\
\hline Largura esquerda & Masculino & $-1,10 \pm 5,69$ & $0,22 \quad 5,35$ & $\mathrm{p}^{(1)}=0,129$ \\
\hline (LRISE) & Feminino & $0,00 \pm 4,85$ & $2,80 \pm 4,47$ & $\mathrm{p}^{(1)}<0,001^{*}$ \\
\hline \multicolumn{2}{|c|}{ Valor de $p$} & $\mathrm{p}^{(1)}=0,185$ & $p^{(1)}=0,001^{*}$ & \\
\hline
\end{tabular}

${ }^{*}$ ): Diferença significante a 5,0\%. (1): Através do teste t-Student com variâncias iguais. (2): Através do teste t-Student com variâncias desiguais.

dos seios maxilares nos dois tipos de radiografia, observou-se ausência de alteração nos indivíduos cadastrados como grupo controle. No grupo experimental verificou-se a presença de alteração na morfologia do seio em 8 indivíduos, estando todas elas relacionadas nos quadrantes inferiores, correspondente às unidades dentárias perdidas.

Os resultados da técnica computadorizada estão dispostos nas Tabelas 4, 5 e 6 . $\mathrm{Na}$ radiografia inicial e após dois anos, a maioria das variáveis as médias foram mais elevadas no sexo masculino do que no feminino.
$\mathrm{Na}$ inicial, comprova-se diferença significante entre os sexos, exceto nas variáveis "Fator de forma direito e esquerdo" no grupo Controle e "Área direita e esquerda" e "Fator de forma direito e esquerdo" e perímetro esquerdo no grupo experimental.

Entre os grupos (controle e experimental), a única diferença significante foi registrada para a variável "Área direita" no sexo feminino, sendo que, neste caso, o valor da média foi mais elevado no grupo experimental do que no grupo controle (Tabela 4).

$\mathrm{Na}$ final, comprovou-se diferença significativa 
Musse JO, Marques JAM, Olibeira RN. Contribuição da análise do seio maxilar para a identificação humana

Tabela 4. Média e desvio padrão das medidas (área, perímetro e fator de forma), segundo o sexo e o grupo na radiografia inicial, São Paulo, 2009

\begin{tabular}{|c|c|c|c|c|}
\hline Variável & Sexo & Grupo Controle | Média \pm DP & Grupo Experimental | Média \pm DP & Valor de $\mathrm{p}$ \\
\hline Área direita & Masculino & $1898,88 \pm 582,02$ & $1870,19 \pm 517,48$ & $\mathrm{p}^{(1)}=0,739$ \\
\hline (BRISD) & Feminino & $1628,47 \pm 427,70$ & $1796,06 \pm 533,14$ & $\mathrm{p}^{(1)}=0,028^{*}$ \\
\hline \multicolumn{2}{|c|}{ Valor de $p$} & $\mathrm{p}^{(1)}=0,001^{*}$ & $\mathrm{p}^{(1)}=0,368$ & \\
\hline Perímetro direito & Masculino & $201,69 \pm 30,18$ & $203,27 \pm 25,43$ & $\mathrm{p}^{(1)}=0,718$ \\
\hline (PRISD) & Feminino & $189,11 \pm 23,55$ & $193,48 \pm 24,59$ & $\mathrm{p}^{(1)}=0,247$ \\
\hline \multicolumn{2}{|c|}{ Valor de $p$} & $\mathrm{p}^{(1)}=0,003^{*}$ & $\mathrm{P}^{(1)}=0,013^{*}$ & \\
\hline Fator de forma & Masculino & $0,58 \pm 0,07$ & $0,57 \pm 0,07$ & $\mathrm{p}^{(1)}=0,883$ \\
\hline direito (FRISD) & Feminino & $0,59 \pm 0,10$ & $0,59 \pm 0,09$ & $\mathrm{p}^{(1)}=0,687$ \\
\hline \multicolumn{2}{|c|}{ Valor de $p$} & $\mathrm{p}^{(1)}=0,372$ & $\mathrm{p}^{(1)}=0,108$ & \\
\hline Área esquerda & Masculino & $2085,39 \pm 571,23$ & $1993,73 \pm 611,94$ & $\mathrm{p}^{(1)}=0,323$ \\
\hline (BRISE) & Feminino & $1745,04 \pm 431,79$ & $1860,42 \pm 553,41$ & $\mathrm{p}^{(1)}=0,139$ \\
\hline \multicolumn{2}{|c|}{ Valor de $p$} & $\mathrm{p}^{(1)}<0,001^{*}$ & $\mathrm{p}^{(1)}=0,145$ & \\
\hline Perímetro esquerdo & Masculino & $213,29 \pm 28,93$ & $207,87 \pm 34,43$ & $\mathrm{p}^{(1)}=0,276$ \\
\hline (PRISE) & Feminino & $196,57 \pm 24,79$ & $199,57 \pm 24,78$ & $\mathrm{p}^{(1)}=0,440$ \\
\hline \multicolumn{2}{|c|}{ Valor de $p$} & $\mathrm{p}^{(1)}<0,001^{*}$ & $\mathrm{p}^{(1)}=0,078$ & \\
\hline Fator de forma & Masculino & $0,57 \pm 0,07$ & $0,57 \pm 0,08$ & $\mathrm{p}^{(1)}=0,845$ \\
\hline esquerdo (FRISE) & Feminino & $0,57 \pm 0,10$ & $0,58 \pm 0,08$ & $\mathrm{p}^{(1)}=0,563$ \\
\hline \multicolumn{2}{|c|}{ Valor de $p$} & $\mathrm{p}^{(1)}=0,914$ & $\mathrm{p}^{(1)}=0,474$ & \\
\hline
\end{tabular}

$\left(^{*}\right)$ : Diferença significante a 5,0\%. (1): Através do teste t-Student com variâncias iguais.

Tabela 5. Média e desvio padrão das medidas (área, perímetro e fator de forma), segundo o sexo e o grupo na radiografia após dois anos de tratamento, São Paulo, 2009

\begin{tabular}{|c|c|c|c|c|}
\hline Variável & Sexo & Grupo Controle | Média \pm DP & Grupo Experimental | Média \pm DP & Valor de $p$ \\
\hline Área direita & Masculino & $1906,55 \pm 501,33$ & $1770,60 \pm 368,90$ & $p(1)=0,049^{*}$ \\
\hline (BRISD) & Feminino & $1705,18 \pm 364,07$ & $1670,47 \pm 457,05$ & $p(2)=0,591$ \\
\hline \multicolumn{2}{|c|}{ Valor de $p$} & $p(2)=0,004^{*}$ & $p(1)=0,125$ & \\
\hline Perímetro direito & Masculino & $203,58 \pm 28,03$ & $195,87 \pm 19,04$ & $p(1)=0,041^{*}$ \\
\hline (PRISD) & Feminino & $189,96 \pm 22,66$ & $187,19 \pm 23,29$ & $p(2)=0,441$ \\
\hline \multicolumn{2}{|c|}{ Valor de $p$} & $p(2)=0,001^{*}$ & $p(2)=0,010^{*}$ & \\
\hline Fator de forma & Masculino & $0,57 \pm 0,07$ & $0,58 \pm 0,07$ & $p(2)=0,525$ \\
\hline direito (FRISD) & Feminino & $0,59 \pm 0,10$ & $0,60 \pm 0,09$ & $p(2)=0,732$ \\
\hline \multicolumn{2}{|l|}{ Valor de $p$} & $p(2)=0,082$ & $p(1)=0,082$ & \\
\hline Área esquerda & Masculino & $2067,89 \pm 548,00$ & $1912,28 \pm 556,20$ & $p(2)=0,073$ \\
\hline (BRISE) & Feminino & $1736,78 \pm 448,95$ & $1780,50 \pm 547,52$ & $p(2)=0,577$ \\
\hline \multicolumn{2}{|c|}{ Valor de $p$} & $\mathrm{p}(2)<0,001^{*}$ & $p(2)=0,128$ & \\
\hline Perímetro esquerdo & Masculino & $212,49 \pm 24,96$ & $201,02 \pm 28,96$ & $p(2)=0,007^{*}$ \\
\hline (PRISE) & Feminino & $194,42 \pm 26,78$ & $192,51 \pm 25,13$ & $p(2)=0,639$ \\
\hline \multicolumn{2}{|l|}{ Valor de $p$} & $\mathrm{p}(2)<0,001^{*}$ & $\mathrm{p}(2)=0,046^{*}$ & \\
\hline Fator de forma & Masculino & $0,57 \pm 0,06$ & $0,58 \pm 0,08$ & $p(2)=0,305$ \\
\hline esquerdo (FRISE) & Feminino & $0,57 \pm 0,10$ & $0,59 \pm 0,09$ & $p(2)=0,199$ \\
\hline \multicolumn{2}{|l|}{ Valor de $p$} & $p(1)=0,682$ & $p(2)=0,355$ & \\
\hline
\end{tabular}

$\left.{ }^{*}\right)$ : Diferença significante a 5,0\%. (1): Através do teste t-Student com variâncias desiguais. (2): Através do teste t-Student com variâncias iguais. 
Musse JO, Marques JAM, Olibeira RN. Contribuição da análise do seio maxilar para a identificação humana

Tabela 6. Média e desvio padrão das diferenças (variação) entre as medidas (área, perímetro e fator de forma) da radiografia inicial e após dois anos de tratamento, segundo o sexo e o grupo, São Paulo, 2009

\begin{tabular}{|c|c|c|c|c|}
\hline Variável & Sexo & Grupo Controle | Média \pm DP & Grupo Experimental | Média \pm DP & Valor de $p^{(1)}$ \\
\hline Área direita & Masculino & $-7,67 \pm 393,06$ & $99,59 \pm 478,41$ & $\mathrm{p}^{(1)}=0,119$ \\
\hline (BRISD) & Feminino & $-76,71 \pm 346,86$ & $125,59 \pm 384,24$ & $\mathrm{p}^{(1)}=0,001^{*}$ \\
\hline \multicolumn{2}{|c|}{ Valor de $p$} & $p^{(1)}=0,235$ & $p^{(1)}=0,702$ & \\
\hline Perímetro direito & Masculino & $-1,89 \pm 19,54$ & $7,40 \pm 23,63$ & $\mathrm{p}^{(1)}=0,007^{*}$ \\
\hline (PRISD) & Feminino & $-0,86 \pm 13,97$ & $6,29 \pm 21,40$ & $\mathrm{p}^{(2)}=0,012^{*}$ \\
\hline \multicolumn{2}{|c|}{ Valor de $p$} & $\mathrm{p}^{(2)}=0,699$ & $\mathrm{p}^{(1)}=0,753$ & \\
\hline Fator de forma & Masculino & $0,01 \pm 0,04$ & $0,00 \pm 0,06$ & $\mathrm{p}^{(2)}=0,272$ \\
\hline direito (FRISD) & Feminino & $-0,01 \pm 0,04$ & $0,00 \pm 0,05$ & $\mathrm{p}^{(1)}=0,906$ \\
\hline \multicolumn{2}{|c|}{ Valor de $p$} & $\mathrm{p}^{(2)}=0,056$ & $\mathrm{p}^{(1)}=0,818$ & \\
\hline Área esquerda & Masculino & $17,50 \pm 583,38$ & $81,44 \pm 444,60$ & $\mathrm{p}^{(1)}=0,431$ \\
\hline (BRISE) & Feminino & $8,25 \pm 223,85$ & $79,91 \pm 334,33$ & $\mathrm{p}^{(2)}=0,109$ \\
\hline \multicolumn{2}{|c|}{ Valor de $p$} & $\mathrm{p}^{(2)}=0,894$ & $\mathrm{p}^{(2)}=0,980$ & \\
\hline Perímetro esquerdo & Masculino & $0,79 \pm 26,90$ & $6,85 \pm 21,79$ & $\mathrm{p}^{(1)}=0,115$ \\
\hline (PRISE) & Feminino & $2,15 \pm 15,80$ & $7,05 \pm 21,82$ & $\mathrm{p}^{(2)}=0,102$ \\
\hline \multicolumn{2}{|c|}{ Valor de $p$} & $\mathrm{p}^{(2)}=0,694$ & $\mathrm{p}^{(1)}=0,951$ & \\
\hline Fator de forma & Masculino & $0,00 \pm 0,06$ & $-0,01 \pm 0,05$ & $p^{(1)}=0,083$ \\
\hline esquerdo (FRISE) & Feminino & $-0,01 \pm 0,06$ & $-0,02 \pm 0,07$ & $\mathrm{p}^{(1)}=0,290$ \\
\hline \multicolumn{2}{|c|}{ Valor de $p$} & $\mathrm{p}^{(1)}=0,450$ & $\mathrm{p}^{(2)}=0,723$ & \\
\hline
\end{tabular}

${ }^{*}$ ): Diferença significante a 5,0\%. (1): Através do teste t-Student com variâncias iguais. (2): Através do teste t-Student.

entre os sexos, com exceção das variáveis fator de forma direito e esquerdo no grupo controle e "áreas direita e esquerda", fator de forma direito e esquerdo no grupo experimental.

Na comparação entre os grupos, observou-se diferença significativa entre os mesmos para as variáveis: "perímetros direito e esquerdo" e "área direita" no sexo masculino (Tabela 5).

A Tabela 6 apresenta a média e o desvio padrão das variações ou diferenças entre os valores encontrados na radiografia inicial e após dois anos de tratamento, por sexo e por grupo.

No grupo experimental, com exceção da variáveis "fator de forma", em ambos os sexos - que apresentaram valores negativos, todas as demais médias foram positivas. Diferenças entre os grupos foram verificadas para "perímetro direito" em ambos os sexos; e "área direita" no sexo feminino. Para estas variáveis observouse que as médias foram positivas no grupo experimental e negativas no grupo controle.

\section{Dıscussão}

Vários métodos são empregados na identificação de restos humanos, sendo a maioria deles baseada na comparação entre dados ante-mortem e post-mortem disponíveis. Embora a técnica da impressão digital seja considerada a mais precisa, em muitos casos, ela não pode ser utilizada, especialmente quando os corpos foram mutilados, decompostos, queimados ou fragmentados ${ }^{13}$.

Neste contexto, a antropometria, através de metodologias qualitativas e quantitativas, vem sendo empregada no processo identificatório, no tocante à investigação e estimativa de dados biotipológicos, como: espécie, sexo, fenótipo cor de pele, idade, estatura e peso ${ }^{14}$.

De acordo com Ribeiro ${ }^{15}$, a assimetria dos seios da face tem estimulado algumas tentativas de identificação geral pela análise de mensurações dos seios obtidas em radiografias. Outros trabalhos utilizam a comparação anatômica ante 
Musse JO, Marques JAM, Olibeira RN. Contribuição da análise do seio maxilar para a identificação humana

e post-mortem na tentativa de conseguir chegar à identificação individual ${ }^{16}$.

Neste estudo, os autores propuseram novos métodos de identificação humana, através de radiografias panorâmicas dos seios maxilares, associando a análise da morfologia dos mesmos com o emprego de métodos quantitativos.

A opção pelo emprego de duas técnicas manuais e outra computadorizada se deu na tentativa de aliar a simplicidade e o baixo custo dos primeiros métodos, passíveis de serem reproduzidos nos IMLs do país que não possuem computadores, à sofisticação do segundo (computadorizado), no qual existe a necessidade de conhecimentos de informática e de aparelhos adequados, além da aquisição do software.

O ImageLab 2000®, software utilizado neste estudo, destina-se a análises morfométricas e subtração de imagens, sendo utilizado para espécimes de escala macroscópica e microscópica, na área de patologia ${ }^{12}$. A utilização deste programa na análise do seio maxilar é justificada pelo fato do mesmo permitir a análise bidimensional da referida estrutura anatômica, fornecendo dados precisos quanto ao cálculo da área, perímetro e fator de forma.

Os seios maxilares foram as estruturas escolhidas para realização desta pesquisa por existirem trabalhos publicados na literatura com características que sugerem a possibilidade de utilização dos mesmos na identificação humana ${ }^{17}$.

Assim, é de consenso entre os autores que os seios maxilares variam de acordo com cada indivíduo e com a idade, e entre os dois seios do mesmo individuo ${ }^{17}$. Além disso, Fernandes ${ }^{8}$ afirma que estas estruturas são maiores nos homens que nas mulheres, sendo o seio maxilar esquerdo mais largo que o direito, em ambos os sexos.

Neste trabalho verificou-se que a maioria das variáveis utilizadas, tanto na técnica manual quanto na computadorizada, foram maiores nos seios maxilares esquerdos e estatisticamente significantes para o sexo, quando analisadas separadamente nas radiografias inicial e após dois anos de tratamento ortodôntico, em ambos os grupos.

Esses achados corroboram com as afirmações de de Silva et al. ${ }^{23}$, segundo as quais a variação de forma, área e simetria dos seios da face constitui uma parâmetro importante para a determinação do dimorfismo sexual, ao contrário do encontrado por Teke et $\mathrm{al}^{10}$.

Entretanto, quando avaliada a variação, ou seja, a diferença entre as medidas das variáveis obtidas nas duas radiografias, obteve-se resultado significante, apenas para largura esquerda, na técnica manual I (grupo experimental), indicando a limitação do emprego da diferença entre as medidas para estimativa do sexo.

Quanto à escolha de pacientes ortodônticos para amostra, esta foi em função da viabilidade de utilização do acervo da clínica de ortodontia. Como grupo experimental, selecionou-se indivíduos que tivessem perdido dentes posteriores superiores envolvidos na área do seio maxilar, sendo estes representados pelos primeiros e segundos pré-molares e primeiros, segundos e terceiros molares superiores direito e esquerdo. Isto porque, de acordo com Tözüm et al. ${ }^{18}$, o assoalho sinusal se estende desde o primeiro pré-molar até a tuberosidade do maxilar.

Em vista da complexidade da anatomia sinusal, diferentes exames radiográficos são necessários para mostrar adequadamente todas as áreas, sendo que as principais radiografias abordadas para esta finalidade são as periapicais, oclusais, panorâmicas, P.A. de Waters, tomografia computadorizada, projeção sub-mento-vertex ou Hirtz, Cadwell ou fronto-naso ${ }^{19}$.

O exame radiográfico mais descrito na literatura para análise dos seios da face tem sido a Tomografia Computadorizada (TC), pois esta oferece boa resolução espacial e elimina a superposição de estruturas pela confecção de imagens seccionais, permitindo, assim, avaliar adequadamente a anatomia dos seios da face e suas freqüentes variações. ${ }^{20}$ Apesar da comprovada eficácia do emprego da TC para análise dos seios da face, optou-se pela panorâmica por esta oferecer uma visão ampla da região maxilo-mandibular e por serem solicitadas com freqüência em diversas especialidades da Odontologia. Gonçalves et al. ${ }^{21}$ avaliaram as solicitações encaminhadas a uma clínica de radiologia durante o referido ano, verificando que a ortodontia foi a especialidade que mais solicitou a radiografia panorâmica, seguida da cirurgia. 
Entretanto, observam-se alguns problemas relativos à utilização da radiografia panorâmica na área forense. Mincer et al. ${ }^{24}$ relataram o fato da maioria dos laboratórios forenses não terem o aparelho e a dificuldade em colocar o corpo em uma posição estática ou sentado, em função da rigidez cadavérica. Conseqüentemente, radiografias panorâmicas pós-morte acabam sendo realizadas transportando os crânios, quando possível, ou realizando adaptações com o aparelho de raio X. Além disso, segundo Wood ${ }^{6}$, a imagem radiográfica de filmes intra-orais ou panorâmicos é influenciada pela quantidade de tecido duro ou mole presente.

Outra informação também relevante é que, muitas vezes, as radiografias não estão devidamente arquivadas, reveladas ou fixadas, limitando sua aplicabilidade quando requisitadas pela justiça. Daí a importância de conscientizar a classe odontológica da necessidade do registro e arquivamento adequado das informações e dados obtidos durante os atendimentos de rotina ${ }^{23}$.

Além da dificuldade técnica e de eventuais problemas com o processamento radiográfico, já mencionados, segundo Antoniazzi ${ }^{24}$, o seio maxilar é a estrutura anatômica que mais pode causar confusão ao olhar menos treinado, quando da interpretação radiográfica. Considerando isto, foi realizada a calibração intra e inter-examinadores, obtendo-se concordância positiva em ambas.

Para Schlickmann et al..25, a determinação da acurácia das medidas antropométricas é problemática, pois o valor correto de qualquer medida é impossível de se conhecer. Portanto, estudos de padronização e confiabilidade de medidas antropométricas minimizam os erros inerentes às variações de técnica de medição antropométrica.

Misch $^{29}$ citou ainda outros fatores que podem alterar a morfologia dos seios maxilares, como perda óssea por doença periodontal, infecções dentárias, pacientes com doença inflamatória crônica, extrações prematuras, entre outros. Segundo Kwak ${ }^{26}$, a perda dentária pode resultar em pneumatização dos seios maxilares, alterando a morfologia dessas estruturas, através da expansão do assoalho sinusal no alvéolo vazio.

No presente trabalho, não foi observada diferença significante entre os grupos controle e experimental para maioria das variáveis. Estes resultados evidenciam a possível aplicabilidade das referidas medidas, para identificação humana pós-morte.

Somado a isto, os resultados da técnica manual II mostraram presença de alteração na morfologia dos seios maxilares nas radiografias de apenas 8 $(2,43 \%)$ participantes, todos estes cadastrados no grupo experimental, ou seja, que tiveram perda dentária no decorrer do tratamento. Se, por um lado, a baixa prevalência de alterações no grupo experimental indica que a perda dentária não foi suficiente para alterar a morfologia dos seios maxilares, por outro, a ausência de alterações no grupo controle sugere que, no período de tempo analisado, a movimentação ortodôntica não funcionou como um agente modificador da anatomia dos seios, reforçando a possibilidade de utilização dos mesmos na área forense.

De acordo com Ajzen et al. ${ }^{27}$, em casos de perda dentária, a reabsorção mais intensa do osso alveolar ocorre imediatamente após a perda dos dentes, resultando em reabsorção e remodelação devido à ausência de carga funcional. A perda óssea vertical no processo alveolar prossegue em aproximadamente $0,1 \mathrm{~mm}$ por ano, podendo variar de indivíduo para indivíduo. Desta forma, o período de tratamento ortodôntico utilizado como intervalo entre as radiografias talvez não tenha sido suficiente para observar alterações morfológicas e métricas significativas nos seios maxilares.

Vale ressaltar que, apesar da ausência de alteração na morfologia dos seios, observouse variação de tamanho dos mesmos entre as radiografias iniciais e finais do tratamento ortodôntico, de um mesmo indivíduo. Para Oliveira et al. ${ }^{28}$ modificações no padrão de estruturas anatômicas de um mesmo indivíduo podem acontecer em função da possibilidade de distorção da imagem nas radiografias panorâmicas, a depender do aparelho utilizado e da incapacidade de ajuste entre a direção da fonte de raios $X$ e áreas específicas do crânio onde ocorrem sobreposições.

\section{ConClusões}

A análise dos seios maxilares mostrou que 
Musse JO, Marques JAM, Olibeira RN. Contribuição da análise do seio maxilar para a identificação humana

a comparação da morfologia e das mensurações destas estruturas em radiografias panorâmicas podem ser empregadas para estimativa de sexo e identificação humana individual.

Os três métodos propostos, tanto os manuais quanto o computadorizado, apresentaram resultados confiáveis, mostrando a aplicabilidade de seu emprego no contexto forense.

A maioria das medidas dos seios maxilares na técnica manual I (altura e largura) e na computado- rizada (área, perímetro e fator de forma) foi significante para investigação do sexo. A comparação radiográfica da morfologia dos seios na técnica manual II demonstrou a contribuição que estas estruturas podem oferecer para identificação humana.

A perda dentária não interferiu na dimensão dos seios maxilares e, conseqüentemente, na identificação humana individual, quando comparados os resultados entre o grupo controle e experimental.

Musse JO, Marques JAM, Olibeira RN. Contribution of the maxillary sinus analysis for human identification, Saúde, Ética \& Justiça. 2009;14(2):65-76.

AвSTRACT: The maxillary sinuses provide important subsidies for forensic identification. The objective of this paper is to evaluate the effectiveness of gender and human identification through maxillary sinus analysis, as well to propose three different identification methods. Its sample was composed by 656 panoramic dental radiographs of 328 individuals from both genders, which were divided by two groups (control and experimental), according to the presence or the absence of tooth loss. Three methods were employed: two manual processes and one generated by computer system. The manual techniques consisted in measuring both height and width from the sinus using acetate sheets, evaluating the morphology of quadrants. The computerized technique used the software IMAGELAB $2000 \circledast$ to calculate sinus' area, perimeter and form factor. Both were followed by a statistical and comparative data analysis. Sinus' morphology and measurement can be used to estimate gender and individual human identification. The analysis of the maxillary sinus associated to other evidences can be used in Forensic Medicine Institutes since it can contribute to human identification studies and cases.

KEY WORDS: Forensic anthropology; Maxillary sinus; Radiography, panoramic; Forensic dentistry.

REFERÊNCIAS

1. Figini $A R L$, Silva JRL, Jobim LF, Silva M. Identificação Humana. São Paulo: Millennium; 2003.

2. Robinson MS, Bidmos MA. The skull and humerus in the determination of sex: reliability of discriminant function equations. Forensic Sci Int. 2009;186(1-3):86-95.

3. Passalacqua NV. Forensic age-at-death estimation from the human sacrum. J Forensic Sci. 2009;54(2):255-62.

4. Oliveira RN, Daruge E, Galvão LCC, Tumang
AJ. Contribuição da odontologia legal à identificação humana post-mortem. Rev Bras Odontol. 1998; 55(2):117-22.

5. Santoro V, Lozito P, Mastrorocco N, Introna F. Morphometric analysis of third molar root development by an experimental method using digital orthopantomographs. J Forensic Sci. 2008;53(4):904-9.

6. Wood RE. Forensic aspects of maxillofacial radiology. Forensic Sci Int. 2006;159(1):4755. 
Musse JO, Marques JAM, Olibeira RN. Contribuição da análise do seio maxilar para a identificação humana

7. Perrella A, Rocha SS, Cavalcanti RGP. Quantitative analyses of maxillary sinus using computed tomography. J Appl Oral Sci. 2003;11(3):229-33.

8. Fernandes CL. Volumetric analysis of maxillary sinuses of Zulu and European crania by helical, multislice computed tomography. J Laryngol Otol. 2004;118(11):877-81.

9. Fernades CL. Forensic ethnic identification of crania: the role of the maxillary sinus - a new approach 2004a;25(4):302-13.

10. Teke HY, Duran S, Canturk N, Canturk G. Determination of gender by measuring the size of maxillary sinuses in computerized tomography scans. Surg Radiol Anat. 2007;29(9):9-13.

11. Ribeiro QAF. Um método de padronização de medidas feitas em radiografias dos seios frontais para ser utilizado na identificação de pessoas [Tese]. São Paulo: Escola Paulista de Medicina; 1993.

12. Novelli MD. A segmentação da imagem histológica digital: novo paradigma para a introdução de uma anatomia digital [Teselivre-docência]. São Paulo: Faculdade de Odontologia da USP; 2002.

13. Kirchhoff S, Fischer F, Lindemaier G, Herzog P, Kirchhoff C, Becker C, et al. Eisenmenger $W$. Is post-mortem CT of the dentition adequate for correct forensic identification?: comparison of dental computed tomograpy and visual dental record. Int J Legal Med. 2008;122(6):471-9.

14. Kalia S, Shetty SK, Patil K, Mahima VG. Stature estimation using odontometry and skull anthropometry. Indian J Dent Res. 2008;19(2):150-4

15. Ribeiro FA. Standardized measurements of radiographic films on frontal sinuses: na AID to identifying Unknown persons. Ear Nose Throat J. 2000;79(1):32-3.

16. Brown KA. Procedures for the collection of dental records for person identification. J Forensic Odontostomatol. 2007;25(2):63-4.

17. Kinsui MM, Guilherme A, Yamashita HK. Variações anatômicas e sinusopatias: estudo por tomografia computadorizada. Rev Bras Otorrinolaringol. 2002; 68(5):64552.

18. Tözüm TF, Dursun E, Tulunoglu I. Sinus floor elevationfromamaxillarymolartoothextraction socket in a patient with chronic inflammation. J Periodontol. 2009;80(3):521-6.

19. Vlijmen OJC, Bergé SJ, Gwen RJS, Bronkhorst EM, Katsaros C, Kuijpers AM. Comparison of cephalometric radiograhs obtained from cone-beam computed tomography scans and conventional radiographs. J Oral Maxillofac Surg. 2009;67:92-7.

20. Cameriere R, Ferrante L, Mirtella D, Rollo FU, Cingolani M. Frontal sinuses for identification: quality of classifications, possible error and potential corrections. J Forensic Sci 2005; 50(4):770-3.

21. Gonçalves A, Gonçalves M, Boscolo FN. Avaliação das solicitações de radiografias recebidas por clínica de radiologia odontológica. Rev Fac Odontol Univ Passo Fundo. 2003;8(1):55-60.

22. Mincer $\mathrm{HH}$, Chaudhry J, Blankenship JA, Tur Ner EW. Postmortem dental radiography. J Forensic Sci. 2008;56(2):405-7.

23. Silva RF, Pereira SDR, Mendes SDSC, Marinho DEA, Júnior ED. Radiografias odontológicas: fonte de informação para identificação humana. Odontologia. Clín. Científ. 2006;5(3):239-42.

24. Antoniazzi MCC, Carvalho PL, Koide $\mathrm{CH}$. Importância do conhecimento da anatomia radiográfica para interpretação de patologias ósseas. RGO. 2008;56(2):195-9.

25. Schlickmann F, Deivis E, Adami F, Guedes V, Assis F. Padronização e confiabilidade das medidas antropométricas para pesquisa 
Musse JO, Marques JAM, Olibeira RN. Contribuição da análise do seio maxilar para a identificação humana

populacional. ALAN. 2007;57(4):335-42.

26. Kwak $\mathrm{HH}$. Topographic anatomy of the inferior wall of the maxillary sinus in koreans. J Oral Maxillofac Surg. 2004;33:382-88.

27. Ajzen SA, Moscatiello RA, Lima MAC, Moscatiello VAM, Moscatiello RM, Nishiguchi $\mathrm{Cl}$, et al. Análise por tomografia computadorizada do enxerto autógeno na cirurgia de "sinus lift" 2005. Radiol Bras. 2005;38(1):25-31.

28. Oliveira MMN, Correia MF, Barata JS. Aspectos relacionados ao emprego da radiografia panorâmica em pacientes infantis. Rev Fac Porto Alegre. 2006; 47:15-9.

29. Misch CE. Implantes dentários contemporâneos. 2a ed. São Paulo: Ed. Santos; 2006.

Recebido em: 27/11/09

Aprovado em: 08/12/09 